\title{
Simultaneous solvent and J-modulation suppression in PGSTE-based diffusion experiments
}

\author{
Davy Sinnaeve*
}

NMR and Structure Analysis Unit, Department of Organic Chemistry, Ghent University, Krijgslaan 281 S4, B-9000 Gent, Belgium

\section{Keywords}

Diffusion; DOSY; PGSTE; WATERGATE; perfect echo; J-modulation; solvent suppression

*Corresponding author.

Address: Krijgslaan 281 S4, B-9000 Gent, Belgium

Phone: +329264 4509

E-mail: Davy.Sinnaeve@UGent.be

\section{Highlights}

- New PGSTE sequences suppressing both solvent resonance and J-modulation

- Employs the perfect echo for scalar coupling refocusing

- Solvent suppression via one or two WATERGATE blocks

- Special attention is given to the robustness of solvent suppression in DOSY

\begin{abstract}
The most favourable solvent suppression methods that have been applied to PGSTE experiments for the measurement of diffusion are WATERGATE and excitation sculpting. However, both methods come with significant J-modulation line-shape distortions on multiplets, a phenomenon that is known to be of particular concern for DOSY data processing. Here, two new PGSTE experiments are proposed that suppress both the solvent peak and Jmodulation based on the perfect echo WATERGATE sequence. This allows narrow suppression bandwidths and thus measurement of diffusion on peaks close to the solvent peak. Both sequences perform admirably and the better option depends on the priority one puts on the quality of the solvent suppression or signal loss due to $\mathrm{T}_{2}$ weighting. Gradient-based solvent suppression in PGSTE experiments can often be compromised by the variable, diffusionencoding gradient pulses. Special emphasis is put on how to maximize the robustness of the solvent suppression.
\end{abstract}

\section{Introduction}

The measurement of molecular translational diffusion in solution by NMR pulsed field gradient (PFG) experiments [1-3] has become an established methodology with a broad range of applications, such as the study of molecular interactions [4-8], spectral editing by diffusion weighting $[9,10]$ or complex mixture analysis under the label of Diffusion Ordered Spectroscopy or DOSY. [8, 11, 12] Diffusion NMR sequences can be divided into two groups, considering whether a spin echo (PGSE, pulsed field gradient spin echo) [13] or stimulated echo (PGSTE, pulsed field gradient stimulated echo) [14] sequence is used. PGSTE has until now been considered as the most versatile of the two, since it suffers much less from scalar 
coupling evolution and, more decisively, it stores the magnetization during the long diffusion delay along the z-axis. For slowly tumbling molecules, where $\mathrm{T}_{1}$ is significantly longer than $\mathrm{T}_{2}$, this leads to the smallest loss of signal even when taking into account the inherent loss of a factor of 2 associated with the STE. In this work, the focus lies on the PGSTE approach. In many cases, the nature of the system under study requires the measurement to be performed under protonated solvent conditions, necessitating appropriate solvent suppression techniques [15]. The most successful solvent suppression sequences that have been incorporated into both PGSE $[16,17]$ and PGSTE $[10,18-20]$ are WATERGATE (WATER suppression by GrAdient-Tailored Excitation) [21] and excitation sculpting [22]. Both techniques apply gradient-enhanced selective echo elements, where only the non-solvent signals are inversed and thus refocused at the end of the sequence. The main advantage of this approach over presaturation-based methods is the retention of signals from protons that are exchangeable with solvent protons, which may be of particular interest to observe in DOSY spectra. [23, 24] All PGSTE sequences integrating WATERGATE or excitation sculpting have until now been applied to bipolar gradient pulse pair sequences (BPPSTE) [25], which has the advantage of suppressing signal-loss due to cross-relaxation or chemical exchange [26, 27]. In the PGSTE-WATERGATE sequence, Price and co-workers $[18,19,28]$ have combined two WATERGATE elements within the bipolar gradient pulse pairs, effectively making them asymmetric, resulting in excellent solvent suppression without phase distortions similar to excitation sculpting. However, for effective diffusion attenuation, this approach relies on the difference between the strengths of the gradient pulses within the asymmetric pair, considerably limiting the available sampling range of gradient strength increments available. An alternative strategy is to simply append the solvent suppression elements to the PGSTE sequence, as implemented with excitation sculpting by Balayssac et al in the BPPSTE-ES sequence [20]. Although this approach recovers the full gradient sampling range, the interference of the variable diffusion encoding gradient strengths with the solvent suppression gradient pulses often leads to refocusing of unwanted solvent coherence transfer pathways (CTPs), resulting in unstable suppression and possible receiver gain overflows.

WATERGATE and excitation sculpting both require selective pulse schemes that have to be up to several milliseconds long to achieve sufficient selectivity. Because of this, all of the above approaches suffer from significant homonuclear scalar coupling evolution (J-modulation) during the sequence, resulting in partial anti-phase multiplets and thus dispersive line-shape contributions. This leads to an increased risk of peak overlap, which is always unfavourable for DOSY processing. Botana et al previously pointed out that line-shape distortions due to Jmodulation are particularly notorious in this regard, since overlap with either the positive or negative tails of the dispersive peak shapes lead to different directions of the 'average' fitted diffusion coefficient, compromising HR-DOSY analysis. [29]

Several strategies to supress J-modulation artefacts have already been applied to PGSE [30-32], BPPSTE [29] and WATERGATE [33] experiments separately. Here, two new BPPSTE sequences are proposed that combine J-modulation and solvent suppression, based on the recently developed "perfect echo" WATERGATE [33]. In addition, some special consideration is given to avoiding distortions on the residual solvent peak that are common in DOSY experiments applying gradient-based solvent suppression. 
(a)

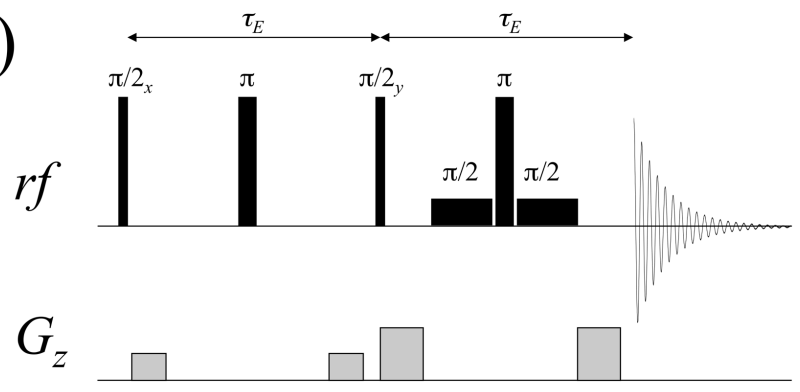

(b)

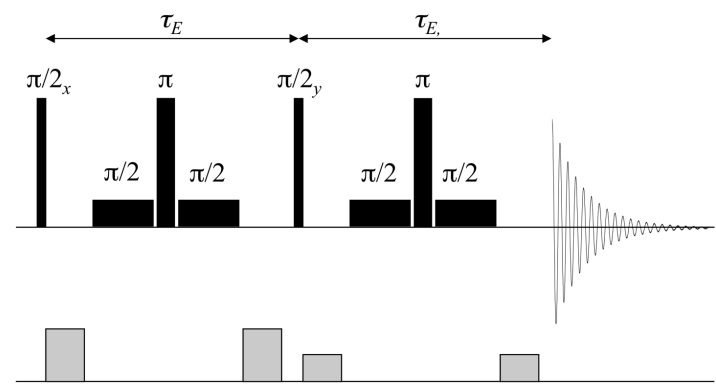

(c)

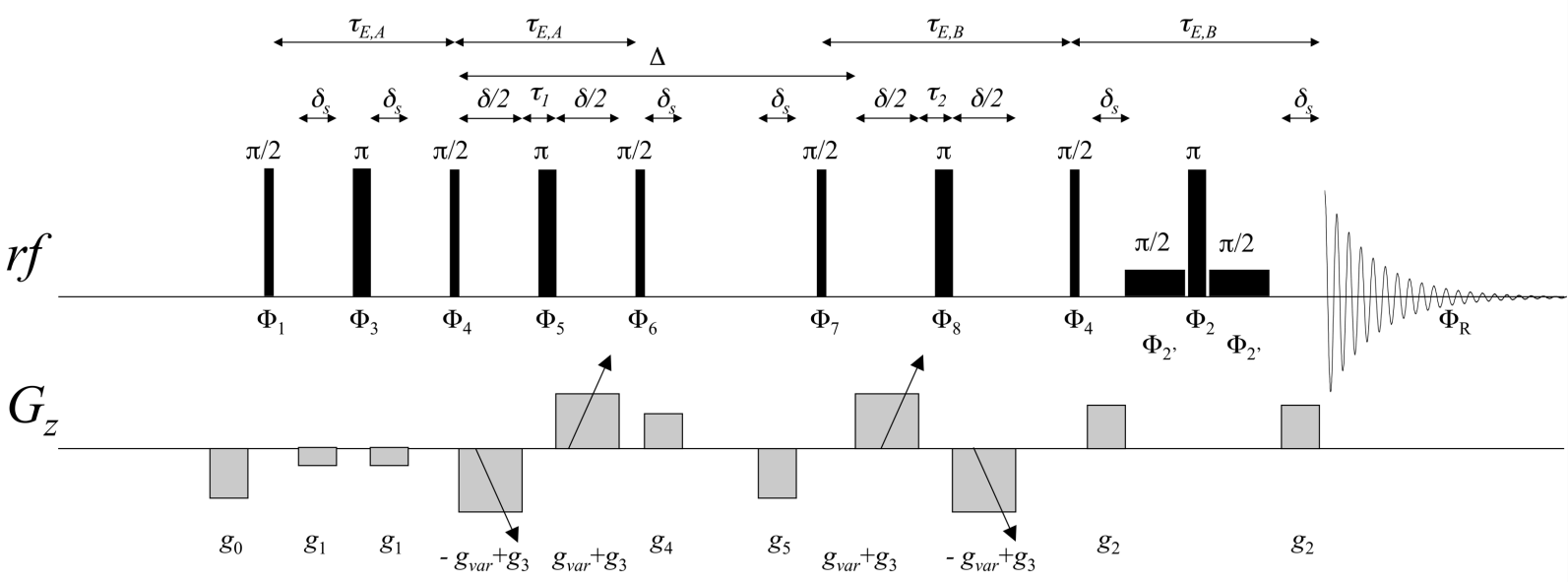

CTP

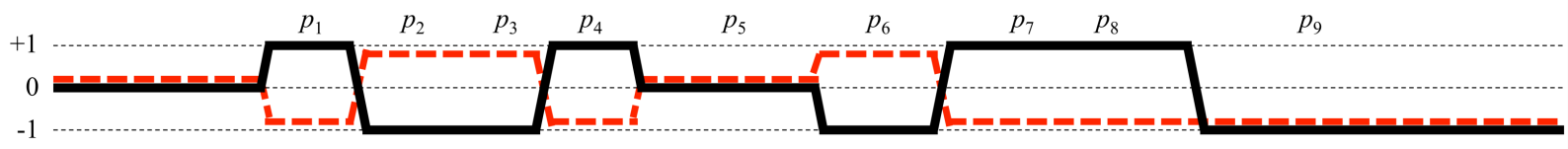

(d)
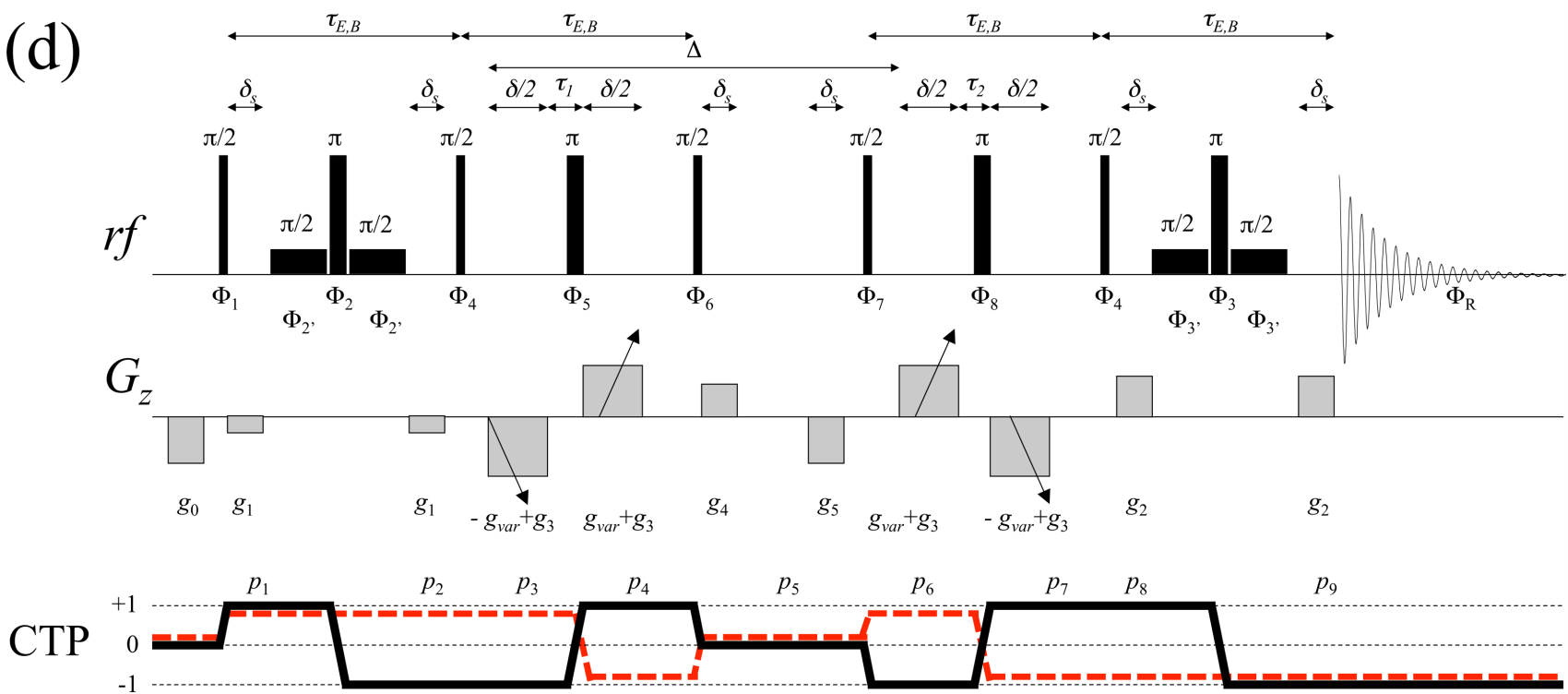

Figure 1: Pulse sequences: (a) PE-WG [33]; (b) PE-ES-WG [33] (c) PE-BPPSTE-WG1; (d) PE-BPPSTE-WG2. Black vertical bars indicate hard rf-pulses with the tilt angle indicated above, black horizontal bars indicate soft rfpulses. One representative desired CTP is shown for both the non-suppressed (black solid line) and suppressed (red dotted line) signals (supplementary material). The gradient strengths, expressed in percentages of their maximum values, are given by $g_{0}=-16 \%, g_{1}=-10 \%, g_{2}=38 \%, g_{3}=-4 \% \times 2 \delta_{\mathrm{s}} / \delta, g_{4}=44 \%$ and $g_{5}=-68 \%$. An alternative set of values to avoid receiver gain overflows is presented in the supplementary material. The gradient scheme is organized such that the ${ }^{2} \mathrm{H}$ lock signal is minimally perturbed. The durations of $\tau_{E, A}$ and $\tau_{E, B}$ depend on which of both sides of their respective perfect echoes requires the most time. The minimum number of transients 
in the phase cycle (Table 1 ) is 16, although increasing to 64 or even 128 transients can still significantly reduce the averaged residual solvent signal intensity (supplementary material).

Table 1: Phase cycle for the PE-BPPSTE-WG sequences presented in Figure 1.

\begin{tabular}{|c|c|}
\hline$\Phi_{1}$ & 0 \\
\hline$\Phi_{2}$ & $01+0_{128} 2_{128}$ \\
\hline$\Phi_{2}{ }^{\prime}$ & $\Phi_{2}+2$ \\
\hline$\Phi_{3}$ & $0_{64} 1_{64}+0_{256} 2_{256}$ \\
\hline$\Phi_{3^{\prime}}$ & $\Phi_{3}+2$ \\
\hline$\Phi_{4}$ & 1 \\
\hline$\Phi_{5}$ & $0_{2} 1_{2}+0_{4} 2_{4} 1_{4} 3_{4}$ \\
\hline$\Phi_{6}$ & $0_{4} 2_{4} 1_{4} 3_{4}+0_{16} 2_{16} 1_{16} 3_{16}$ \\
\hline$\Phi_{7}$ & $0_{4} 2_{4} 1_{4} 3_{4}+0_{16} 2_{16} 1_{16} 3_{16}$ \\
\hline$\Phi_{8}$ & $0_{16} 2_{16} 1_{16} 3_{16}$ \\
\hline$\Phi_{R}$ & $2 \Phi_{2}+2 \Phi_{3}-2 \Phi_{5}+\Phi_{6}+\Phi_{7}-2 \Phi_{8}$ \\
\hline
\end{tabular}

The phases are represented as multiples of $90^{\circ}$, i.e. $0=0^{\circ}, 1=90^{\circ}, 2=180^{\circ}, 3=270^{\circ}$. Other numbers resulting from the algebraic expressions are congruent to the aforementioned numbers (modulo 4). Subscripts represent repetition of a phase. Plus and minus signs indicate addition and subtraction of the phases at matching positions in the series, for which each series must first be repeated until uniform series length is reached. The fully expanded phase cycle is provided in the supplementary material.

\section{Pulse sequences}

J-modulation line-shape artefacts can be minimised by means of a perfect echo (PE) element [34]. This consists out of two individual spin-echoes of duration $\tau_{E}$ with in-between a $90^{\circ}$ pulse possessing a phase quadrature to the excitation pulse, thus only affecting the antiphase magnetization developed during the first spin echo. It can be shown that at the end of the perfect echo, the homonuclear scalar coupling evolution is perfectly refocused within an AX spin system [34], while for arbitrary spin systems this occurs equally well when the condition $J \tau_{E} / 2 \ll 1$ is satisfied and the system was initially at equilibrium [35]. Recently, a perfect echo version of WATERGATE was developed (PE-WG), achieving similar solvent suppression properties, but removing practically all J-modulation effects (Figure 1a). [33] In a similar strategy as for excitation sculpting (ES), a second WATERGATE block can be incorporated into the perfect-echo (PE-ES-WG), further increasing solvent suppression efficiency (Figure 1b). Contrary to true excitation sculpting though, the perfect echo requires the individual WATERGATE blocks to adhere to particular symmetry properties to ensure chemical shift refocusing at the quadrature pulse. [22, 33]

Figure 1 shows two BPPSTE sequences wherein the spin echoes accommodating the bipolar pulse pair gradients both have been recruited as halves of perfect echo elements. These sequences thus achieve scalar coupling refocusing on both sides of the STE sequence. Note that only the second half of the first PE and the first half of the second PE can be used for diffusion encoding, as the quadrature pulse does not tolerate the solute magnetization to be dephased. The PE-BPPSTE-WG1 sequence (Figure 1c) recuperates the second half of the second perfect echo as a WATERGATE block, delivering solvent suppression of similar quality as the PE-WG sequence. In the PE-BPPSTE-WG2 sequence, a second WATERGATE block is incorporated into the first PE, providing similar solvent suppression properties as PE-ES-WG.

A notable difference to the BPPSTE-ES sequence [20] is the absence of a longitudinal eddy current delay (LED), [36] which would have complicated the sequence and thus proper coherence selection (vide infra) even further by introducing additional rf-pulse intervals and 
gradient pulses. This exclusion is warranted on modern-day probes with shielded gradient coils applying moderate gradient strengths.

Both sequences apply asymmetric bipolar gradient pairs for increased coherence selection similarly as in the Oneshot DOSY sequence [37]. Contrary to the Oneshot however, the difference in gradient strength within the bipolar pulse pair is kept constant $\left(2 g_{3}\right)$ rather than the ratio. This approach was preferred here as it allows more robust solvent peak suppression (vide infra). Additionally, it generally compromises less the maximum attainable value for the diffusion encoding variable gradient strength $g_{v a r}$. The constant gradients do cause the StejskalTanner equation to deviate slightly from its standard Gaussian form:

$$
\begin{gathered}
S=S_{0} e^{-D \gamma^{2} \delta^{2} \sigma^{2} g_{v a r}^{2}\left(\Delta+\frac{2(\kappa-\lambda)-1}{4} \delta-\frac{3 \tau_{1}-\tau_{2}}{4}+\frac{g_{3}^{2}}{g_{v a r}^{2}}\left(\frac{2(\kappa-\lambda)+1}{4} \delta+\frac{\tau_{1}+\tau_{2}}{4}\right)-\frac{g_{3}}{g_{v a r}}\left(\frac{\delta}{2}+\frac{\tau_{1}+\tau_{2}}{2}\right)\right)} \\
=S_{0}^{\prime} e^{-D \gamma^{2} \delta^{2} \sigma^{2} g_{v a r}^{2}\left(\Delta+\frac{2(\kappa-\lambda)-1}{4} \delta-\frac{3 \tau_{1}-\tau_{2}}{4}-\frac{g_{3}}{g_{v a r}}\left(\frac{\delta}{2}+\frac{\tau_{1}+\tau_{2}}{2}\right)\right)}
\end{gathered}
$$

Here, $\Delta$ is the diffusion delay, $\delta$ is the total duration of the gradient pulses within a bipolar pair, $\tau_{1}$ and $\tau_{2}$ are the intergradient delays within respectively the first and second bipolar pair, $g_{\text {var }}$ and $g_{3}$ are the gradient strengths as defined in Figure 1, $D$ is the diffusion coefficient, $\gamma$ the gyromagnetic ratio and $S_{0}^{\prime}$ the pre-exponential factor that is independent on $g_{v a r}$ if $g_{3}$ does not relate to $g_{v a r}$. The parameters $\sigma, \kappa$ and $\lambda$ solely depend on the gradient pulse shape applied, e.g. $\sigma=1, \kappa=1 / 3$ and $\lambda=1 / 2$ for a rectangular shape, while for a sine bell shape $\sigma=2 / \pi$, $\kappa=3 / 8$ and $\lambda=1 / 2$. [38] The non-Gaussian behaviour has the minor, non-critical drawback that a modified gradient increment scheme needs to be calculated when there are requirements in this respect. This is the case when using the DECRA processing method, which requires the exponential decay to be linearly sampled. [39]

\section{Pulse sequence performance}

Figure 2 demonstrates the performance of both PE-BPPSTE-WG sequences relative to regular WATERGATE, a double WATERGATE sequence [22], PE-WG and PE-ES-WG. The sample used is a 3\% solution in 90:10 $\mathrm{H}_{2} \mathrm{O}: \mathrm{D}_{2} \mathrm{O}$ of Fristi ${ }^{\circledR}$, a yoghurt-based sweet drink. All J-modulation lineshape artefacts are removed as effectively as in the PE-WG spectra. The quality and bandwidths of the solvent suppression are comparable to those of the PE-WG and PE-ES-WG sequences. In general, the PE-BPPSTE-WG2 sequence provides the cleanest solvent suppression and the least distorted baselines. Double WATERGATE blocks do come at the expense of a less narrow suppression bandwidth relative to single WATERGATE blocks for the same soft pulse length. [22] In addition, given the same total soft pulse lengths, the PEBBPSTE-WG2 sequence inherently cannot achieve as narrow suppression bandwidths compared to methods based on true excitation sculpting, such as PGSTE-WATERGATE and BPPSTE-ES. This is due to the symmetry requirements of the WATERGATE blocks within the perfect echoes, where it is true excitation sculpting's main feature to be relieved of this constraint. [18, 22] However, just as in PE-WG [33], the perfect echoes allow the suppression bandwidth to be significantly narrowed down by increasing the soft pulse lengths without introducing severe J-modulation line-shape artefacts, more than compensating the disadvantage of PE-BPPSTE-WG2 compared to true excitation sculpting.

Figure 3 illustrates the performance of the PE-BPPSTE-WG2 sequence at various soft pulse lengths. At $10.5 \mathrm{~ms} 90^{\circ}$ soft pulse length, the lactose and glucose signals located merely $0.1 \mathrm{ppm}(50 \mathrm{~Hz})$ next to the residual water peak can be detected with sufficient sensitivity to 
be included into a DOSY analysis. The PE-BPPSTE-WG sequences are thus particularly useful when peaks nearby the solvent frequency are of interest. Increasing the soft pulse length does come at the price of increasing the perfect echo durations and thus $\mathrm{T}_{2}$ weighting. For this reason, PE-BPPSTE-WG1 may be the preferred choice in situations where this is a concern, as in this case only one of the perfect echoes has to be matched to the soft pulse lengths.

Additionally, at very long soft pulse lengths, the perfect echo assumption $J \tau_{E} / 2 \ll 1$ may no longer be satisfied, resulting in the reintroduction of some J-modulation distortions.
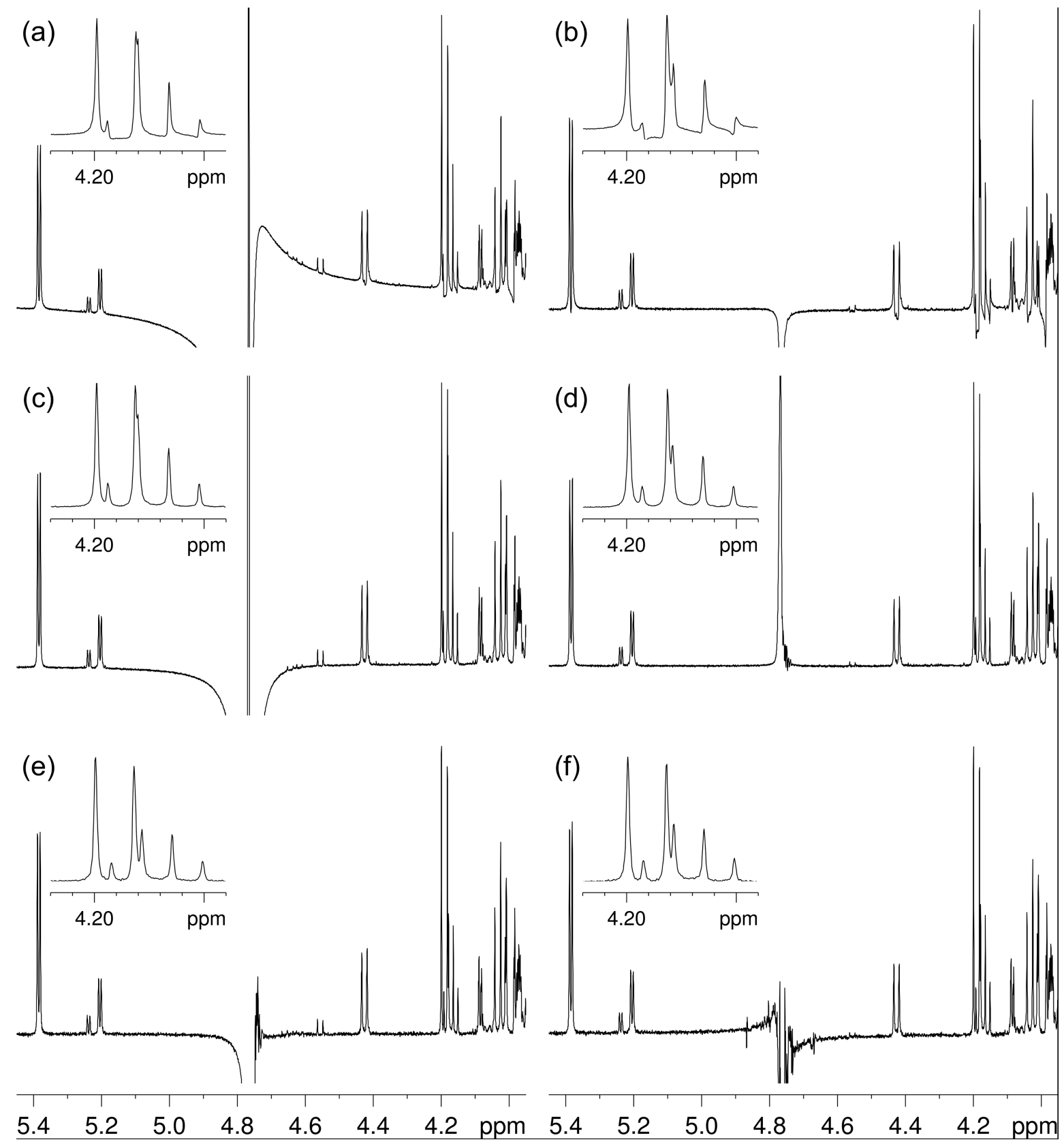

Figure 2: 1D ${ }^{1} \mathrm{H}$ spectra (selected region) of a dilution of Fristi $^{\circledR}$, applying regular WATERGATE (a), a double WATERGATE sequence (b), PE-WG (c), PE-ES-WG (d), PE-BPPSTE-WG1 (e) and PE-BPPSTE-WG2 (f). The number of transients was 16 and the $90^{\circ}$ soft pulse length was $4 \mathrm{~ms}$ throughout. For (e) and (f), $\delta s=0.8 \mathrm{~ms}, \delta=2.5 \mathrm{~ms}$, $\Delta=150 \mathrm{~ms}$ and $g_{\text {var }}$ was set to $10 \%$ of the maximum gradient strength. Severe J-modulation artefacts can be seen in (a) and (b), while this is suppressed in (c) to (f). This is further illustrated in the insets, which show a sucrose doublet and a lactate quadruplet overlapping with each other. 

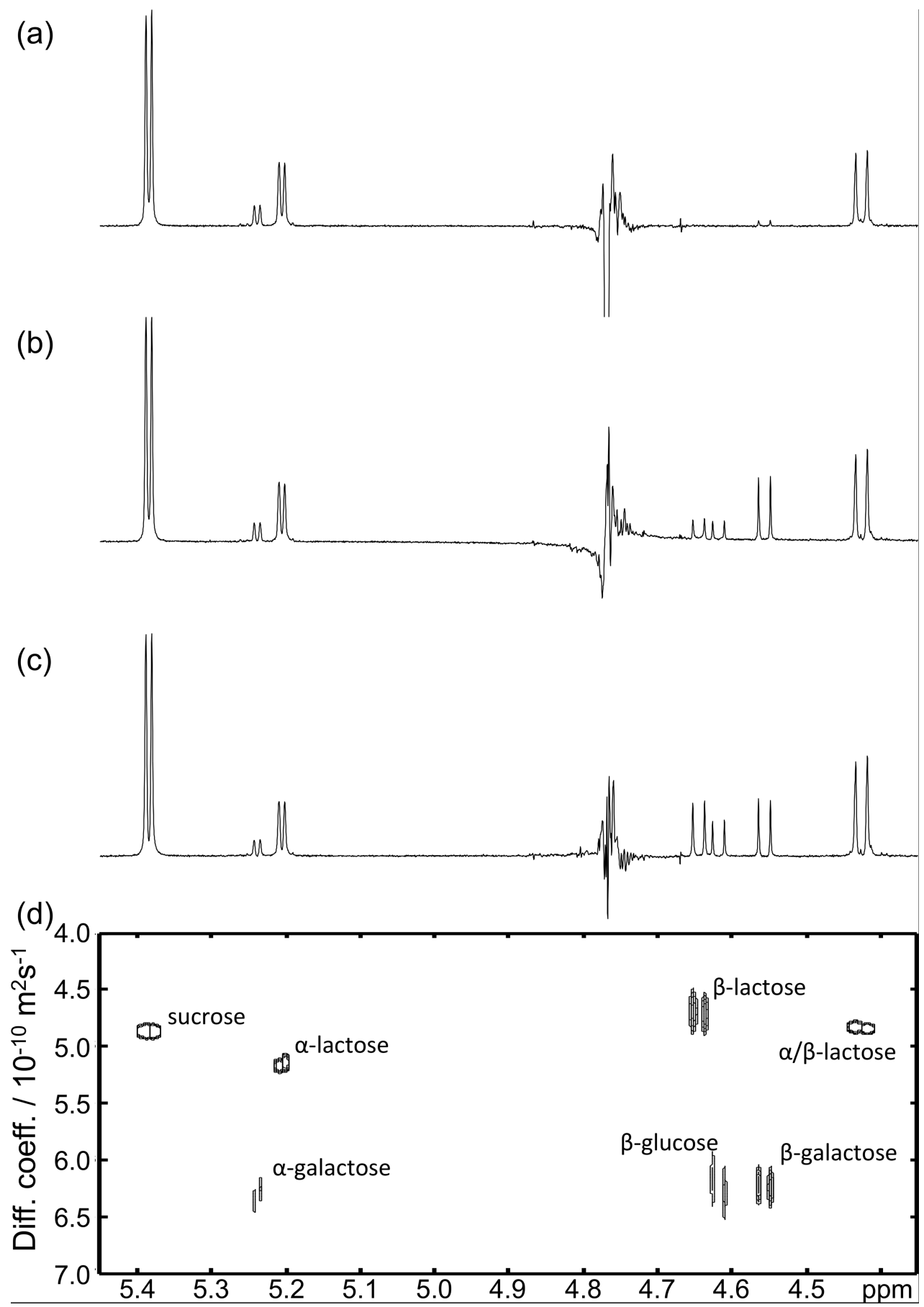

Figure 3: Anomeric proton resonance region of Frist $^{\circledR}$, applying the PE-BPPSTE-WG2 sequence averaged over 128 transients, with soft pulse lengths of $4 \mathrm{~ms}$ (a), $8 \mathrm{~ms}$ (b) and $10.5 \mathrm{~ms}$ (c). Delays and gradient strengths were set as indicated in Figure 2. At $10.5 \mathrm{~ms}$ soft pulse length, the suppression bandwidth is sufficiently narrow to detect the $\beta$-lactose and $\beta$-glucose anomeric proton resonances. Under these conditions, 32 increments were measured with $g_{v a r}$ ranging linearly between $2 \%$ and $95 \%$. With the DOSYToolbox v2.0, [40] a DOSY plot was generated from this dataset as shown in (d), demonstrating the feasibility to measure with these sequences diffusion on resonances very close to the solvent resonance. A selected number of signal intensity decays are included in the supplementary material. Peak assignments were made by comparison to reference spectra in the Human Metabolome Database. [41] 


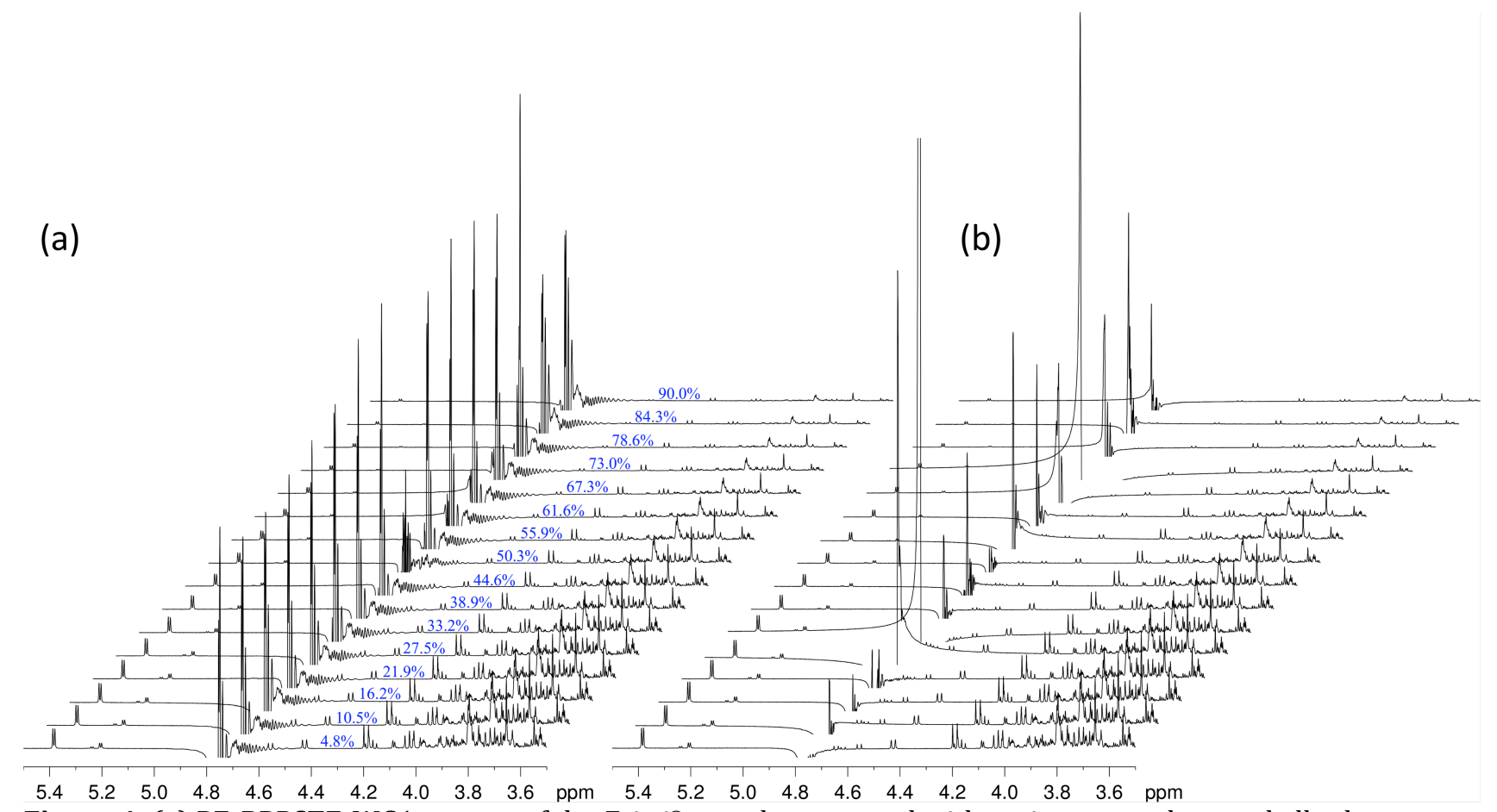

Figure 4: (a) PE-BPPSTE-WG1 spectra of the Fristi ${ }^{\circledR}$ sample measured with various $g_{\text {var }}$ values and all other parameters set as described in Figure 2. The soft pulse length was $8 \mathrm{~ms}$. Averaging was performed over 16 transients, using two different phase cycles for comparison: (a) $\Phi_{5}=0213, \Phi_{6}=\Phi_{7}=0213+0{ }_{4} 2_{4} 1_{4} 3_{4}$ and $\Phi_{7}=$ $0_{4} 2_{4} 1_{4} 3_{4}$; (b) $\Phi_{1}=0_{8} 2_{8}, \Phi_{6}=0213+0_{4} 2_{4}, \Phi_{7}=0213$. The phase cycle strategy of (a) clearly leads to a superior robustness of the residual solvent peak towards the value of $g_{v a r}$.

\section{Robustness of solvent suppression within PGSTE experiments}

Integrating gradient-based solvent suppression schemes in PGSTE sequences is complicated by the presence of the diffusion encoding variable gradients. In general, undesired CTPs arising from rf-pulse imperfections can be suppressed through spoil gradients. However, the degree of dephasing that these CTPs possess at the end of the sequence depends on the variable gradient strength, possibly leading to incidental refocusing. For solute peaks, this typically results in relatively minor gradient-dependent signal contributions. However, for the strong solvent signal, these contributions can lead to significant intensity and line-shape artefacts of its residual signal, resulting in a seemingly erratic behaviour as $g_{v a r}$ varies. This substantially disrupts the spectrum baseline around the solvent peak, affecting the usefulness of the DOSY spectrum for nearby solute peaks and effectively disrupting the solvent suppression quality. Because of the solvent peak's strong dependence on $g_{v a r}$, it is very hard or even impossible to design an adequate stand-alone spoil gradient scheme. Therefore, even more so than in DOSY without solvent suppression, sufficient signal averaging applying a proper phase cycle is a necessity to obtain good quality spectra with robust residual solvent peak behaviour and therefore warrants some attention. All of this is especially true when the sequence contains many rf-pulse intervals containing gradient pulses, resulting in a high number of possible undesired CTPs. This is effectively the case for the PE-BPPSTE-WG sequences, where the gradient-based CTP selection criterion is given by (assuming for simplicity equal values for $\delta_{s}$ and $\delta / 2$ ):

$$
\begin{gathered}
p_{1} g_{1}+p_{2} g_{1}+p_{3}\left(-g_{v a r}+g_{3}\right)+p_{4}\left(g_{v a r}+g_{3}\right)+p_{5}\left(g_{4}+g_{5}\right)+p_{6}\left(g_{v a r}+g_{3}\right) \\
+p_{7}\left(-g_{v a r}+g_{3}\right)+p_{8} g_{2}+p_{9} g_{2}=0
\end{gathered}
$$


To achieve a solvent suppression that is as robust as possible, the dependence on $g_{v a r}$ should be removed from the above equation. Assuming the solvent resonance's maximum coherence order is $1\left(|p|_{\max }=1\right)$, this can be achieved by introducing a 16 step phase cycle that selects the conditions $p_{3}=p_{6}$ and $p_{4}=p_{7}$ (Appendix A). Combining these with the gradient-based criterion leaves the overall CTP selection without any dependence on $g_{v a r}$ :

$$
p_{1} g_{1}+p_{2} g_{1}+2 p_{3} g_{3}+2 p_{4} g_{3}+p_{5}\left(g_{4}+g_{5}\right)+p_{8} g_{2}+p_{9} g_{2}=0
$$

Note that if a constant gradient strength ratio rather than a constant difference within the bipolar pulse pair was chosen (vide supra), a dependence on $g_{v a r}$ would still have been present through $g_{3}$. From this point on, the remaining CTP selection can rely much more robustly on the choice of spoil gradient values. Figure 4 compares the performance of this phase cycling strategy with an alternative one, illustrating the importance of phase cycling to eliminate erratic residual solvent peak behaviour. It should be noted that phase cycling is in essence a spectral subtraction method, and thus its effectiveness in this respect is limited by factors such as the quality of spectrometer's field and lock stability. This phase cycling strategy is embedded into the one proposed in Table 1 and is achieved at 64 transients (see the supplementary material for a more extended rationalisation of the full phase cycle).

Besides those arising from rf-pulse imperfections, there are also undesired CTPs that would get through even when the rf-pulses would be ideal. When these are refocused, their contribution to the residual solvent signal is typically very strong, with a high probability of receiver gain overflow. Fortunately, the $g_{v a r}$ values where these may occur are predictable (supplementary material). One solution is then to simply avoid these critical $g_{v a r}$ values, or discard the affected increments after the experiment. A more elegant solution is to swap the spoil gradient values with an alternative set that lead to different critical $g_{v a r}$ values. This approach was implemented for the PE-BPPSTE-WG sequences, further improving the robustness of their solvent suppression (supplementary material). Note that changing spoil gradients can have a very slight effect on the $S_{0}$ term of the Stejskal-Tanner equation due to altering the diffusion signal-loss caused by these. This can in principle be taken into account during data processing, although in practice the effect should be negligible.

\section{Conclusions}

The PE-BPPSTE-WG sequences allow diffusion measurements combined with high quality solvent and J-modulation suppression, the latter of which can complicate DOSY analysis. Narrow suppression bandwidths are achievable, allowing the study of solute peaks nearby the residual solvent resonance. The PE-BPPSTE-WG1 sequence is preferred when narrow suppression bandwidths combined with minimal signal loss due to $\mathrm{T}_{2}$ weighting is needed, while the PE-BPPSTE-WG2 sequence generally results in cleaner solvent suppression results. These sequences will be particularly useful for systems that must be studied under protonated solvent conditions and where diffusion weighting or analysis is required, such as complex biological fluids or protein-ligand interactions.

\section{Experimental}


All measurements were performed on a Bruker Avance III spectrometer operating at a ${ }^{1} \mathrm{H}$ frequency of $500.13 \mathrm{MHz}$ and equipped with a BBI-Z probe with a calibrated maximum gradient strength of $50.2 \mathrm{G} \mathrm{cm}^{-1}$. Smoothed rectangle gradient pulse shapes were used throughout. Measurements were performed on Fristi ${ }^{\circledR}$, a dairy-based food beverage made from yoghurt, sweetened with several sugar additives (FrieslandCampina N.V.). The sample was prepared by diluting $18 \mu \mathrm{l}$ Fristi with $60 \mu \mathrm{l}$ of $\mathrm{D}_{2} \mathrm{O}$ and $522 \mu \mathrm{l} \mathrm{H} \mathrm{H}_{2} \mathrm{O}$. Sample temperature was set at $25^{\circ} \mathrm{C}$. Bruker pulse programs and details on how to set up the experiments are provided as supplementary material. 2D DOSY processing was performed using the standard univariate method in the DOSY Toolbox [40] version 2.0 running under MATLAB 8.0.0783.

\section{Appendix A}

Within the desired CTP, the coherence orders during the pulse intervals containing the variable gradients in the PE-BPPSTE-WG sequences are related as $p_{3}-p_{6}=0$ and $p_{4}-p_{7}=0$. The difference in coherence order between two delays $\Delta p_{i, j}$ can be controlled by phase cycling all rf-pulses between those pulse intervals simultaneously. The CTP selection condition is then $\Delta p_{i, j}=\Delta q \pm 4 n$ for a 4-step phase cycle, where $n=0,1,2, \ldots$ and $\Delta q$ is the desired coherence order difference that is imposed by the implementation of the phase cycle in the receiver

phase. Assuming the solvent has a $|p|_{\text {max }}=1$, thus implying $\left|\Delta p_{i, j}\right|_{\text {max }}=2$, and choosing $\Delta q=0$, a 4-step phase cycle will limit the coherence jump to just one possibility: $\Delta p_{i, j}=0$. Therefore, two 4-step phase cycles (16 steps in total) implemented as $\Phi_{5}=0213, \Phi_{6}=\Phi_{7}=$ $0213+0_{4} 2_{4} 1_{4} 3_{4}$ and $\Phi_{7}=0_{4} 2_{4} 1_{4} 3_{4}$ imposes the conditions $\Delta p_{3,6}=0$ and $\Delta p_{4,7}=0$ on the solvent coherence.

\section{Acknowledgements}

I thank Prof. Gareth A. Morris from the University of Manchester for stimulating discussions. The Fund for Scientific Research-Flanders (FWO-Vlaanderen) is acknowledged for a postdoctoral mandate.

\section{References}

[1] W.S. Price, NMR Studies of Translational Motion, Cambridge University Press, Cambridge, 2009.

[2] G.A. Morris, Diffusion-ordered spectroscopy (DOSY), in: D.M. Grant, R.K. Harris (Eds.) Encyclopedia of Nuclear Magnetic Resonance, John Wiley \& Sons Ltd., Chichester, UK, 2002, pp. 35-44.

[3] C.S. Johnson, Diffusion ordered nuclear magnetic resonance spectroscopy: principles and applications, Prog. Nucl. Magn. Reson. Spectrosc., 34 (1999) 203-256.

[4] D. Sinnaeve, P.M.S. Hendrickx, J. Van hemel, E. Peys, B. Kieffer, J.C. Martins, The Solution Structure and Self-Association Properties of the Cyclic Lipodepsipeptide Pseudodesmin A Support Its Pore-Forming Potential, Chem.-Eur. J., 15 (2009) 12653-12662.

[5] M. Schonhoff, NMR studies of sorption and adsorption phenomena in colloidal systems, Curr Opin Colloid In, 18 (2013) 201-213.

[6] L.H. Lucas, C.K. Larive, Measuring ligand-protein binding using NMR diffusion experiments, Concept. Magn. Res. Part A, 20A (2004) 24-41. 
[7] Z. Hens, J.C. Martins, A Solution NMR Toolbox for Characterizing the Surface Chemistry of Colloidal Nanocrystals, Chem Mater, 25 (2013) 1211-1221.

[8] Y. Cohen, L. Avram, L. Frish, Diffusion NMR spectroscopy in supramolecular and combinatorial chemistry: An old parameter - New insights, Angew. Chem. Int. Edit., 44 (2005) 520-554.

[9] L.H. Lucas, C.K. Larive, P.S. Wilkinson, S. Huhn, Progress toward automated metabolic profiling of human serum: Comparison of CPMG and gradient-filtered NMR analytical methods, Journal of Pharmaceutical and Biomedical Analysis, 39 (2005) 156-163.

[10] M.L. Liu, J.K. Nicholson, J.C. London, High-resolution diffusion and relaxation edited oneand two-dimensional ${ }^{1} \mathrm{H}$ NMR spectroscopy of biological fluids, Anal. Chem., 68 (1996) 33703376.

[11] D.Y. Li, I. Keresztes, R. Hopson, P.G. Williard, Characterization of Reactive Intermediates by Multinuclear Diffusion-Ordered NMR Spectroscopy (DOSY), Acc. Chem. Res., 42 (2009) 270280.

[12] N. Giuseppone, J.L. Schmitt, L. Allouche, J.M. Lehn, DOSY NMR experiments as a tool for the analysis of constitutional and motional dynamic processes: Implementation for the driven evolution of dynamic combinatorial libraries of helical strands, Angew. Chem. Int. Edit., 47 (2008) 2235-2239.

[13] E.O. Stejskal, J.E. Tanner, Spin diffusion measurements: spin echoes in the presence of a time-dependent field gradient, J Chem Phys, 42 (1965) 288-292.

[14] J.E. Tanner, Use of Stimulated Echo in NMR-Diffusion Studies, J Chem Phys, 52 (1970) 2523-2526.

[15] G. Zheng, W.S. Price, Solvent signal suppression in NMR, Prog. Nucl. Magn. Reson. Spectrosc., 56 (2010) 267-288.

[16] W.S. Price, F. Elwinger, C. Vigouroux, P. Stilbs, PGSE-WATERGATE, a new tool for NMR diffusion-based studies of ligand-macromolecule binding, Magn Reson Chem, 40 (2002) 391395.

[17] K.I. Momot, P.W. Kuchel, Convection-compensating PGSE experiment incorporating excitation-sculpting water suppression (CONVEX), J Magn Res, 169 (2004) 92-101.

[18] G. Zheng, T. Stait-Gardner, P.G.A. Kumar, A.M. Torres, W.S. Price, PGSTE-WATERGATE: An STE-based PGSE NMR sequence with excellent solvent suppression, J Magn Res, 191 (2008) 159-163.

[19] G. Zheng, W.S. Price, Simultaneous convection compensation and solvent suppression in biomolecular NMR diffusion experiments, J Biomol NMR, 45 (2009) 295-299.

[20] S. Balayssac, M.A. Delsuc, V. Gilard, Y. Prigent, M. Malet-Martino, Two-dimensional DoSY experiment with Excitation Sculpting water suppression for the analysis of natural and biological media, J Magn Res, 196 (2009) 78-83.

[21] M. Piotto, V. Saudek, V. Sklenar, Gradient-Tailored Excitation for Single-Quantum NMRSpectroscopy of Aqueous-Solutions, J Biomol NMR, 2 (1992) 661-665.

[22] T.L. Hwang, A.J. Shaka, Water Suppression That Works - Excitation Sculpting Using Arbitrary Wave-Forms and Pulsed-Field Gradients, J Magn Res Ser A, 112 (1995) 275-279. [23] T. Brand, E.J. Cabrita, G.A. Morris, R. Gunther, H.J. Hofmann, S. Berger, Residue-specific NH exchange rates studied by NMR diffusion experiments, J Magn Res, 187 (2007) 97-104. [24] E.J. Cabrita, S. Berger, HR-DOSY as a new tool for the study of chemical exchange phenomena, Magn Reson Chem, 40 (2002) S122-S127.

[25] D.H. Wu, A.D. Chen, C.S. Johnson, An Improved Diffusion-Ordered Spectroscopy Experiment Incorporating Bipolar-Gradient Pulses, J Magn Res Ser A, 115 (1995) 260-264. [26] A.D. Chen, C.S. Johnson, M. Lin, M.J. Shapiro, Chemical exchange in diffusion NMR experiments, J. Am. Chem. Soc., 120 (1998) 9094-9095. 
[27] M.D. Pelta, H. Barjat, G.A. Morris, A.L. Davis, S.J. Hammond, Pulse sequences for highresolution diffusion-ordered spectroscopy (HR-DOSY), Magn Reson Chem, 36 (1998) 706-714. [28] G. Zheng, W.S. Price, Direct Hydrodynamic Radius Measurement on Dissolved Organic Matter in Natural Waters Using Diffusion NMR, Environ Sci Technol, 46 (2012) 1675-1680. [29] A. Botana, J.A. Aguilar, M. Nilsson, G.A. Morris, J-modulation effects in DOSY experiments and their suppression: The Oneshot45 experiment, J Magn Res, 208 (2011) 270-278.

[30] A.M. Torres, G. Zheng, W.S. Price, J-compensated PGSE: an improved NMR diffusion experiment with fewer phase distortions, Magn Reson Chem, 48 (2010) 129-133.

[31] A.M. Torres, R. Dela Cruz, W.S. Price, Removal of J-coupling peak distortion in PGSE experiments, J Magn Res, 193 (2008) 311-316.

[32] J.A. Aguilar, R.W. Adams, M. Nilsson, G.A. Morris, Suppressing exchange effects in diffusionordered NMR spectroscopy, J Magn Res, 238 (2014) 16-19.

[33] R.W. Adams, C.M. Holroyd, J.A. Aguilar, M. Nilsson, G.A. Morris, "Perfecting" WATERGATE: clean proton NMR spectra from aqueous solution, Chem. Commun., 49 (2013) 358-360.

[34] K. Takegoshi, K. Ogura, K. Hikichi, A Perfect Spin-Echo in a Weakly Homonuclear J-Coupled Two Spin-1/2 System, J Magn Res, 84 (1989) 611-615.

[35] J.A. Aguilar, M. Nilsson, G. Bodenhausen, G.A. Morris, Spin echo NMR spectra without J modulation, Chem. Commun., 48 (2012) 811-813.

[36] S.J. Gibbs, C.S. Johnson, A PFG NMR Experiment for Accurate Diffusion and Flow Studies in the Presence of Eddy Currents, J Magn Res, 93 (1991) 395-402.

[37] M.D. Pelta, G.A. Morris, M.J. Stchedroff, S.J. Hammond, A one-shot sequence for highresolution diffusion-ordered spectroscopy, Magn Reson Chem, 40 (2002) S147-S152.

[38] D. Sinnaeve, The Stejskal-Tanner equation generalized for any gradient shape-an overview of most pulse sequences measuring free diffusion, Concept. Magn. Res. Part A, 40A (2012) 39-65.

[39] W. Windig, B. Antalek, Direct exponential curve resolution algorithm (DECRA): A novel application of the generalized rank annihilation method for a single spectral mixture data set with exponentially decaying contribution profiles, Chemometr. Intell. Lab., 37 (1997) 241-254. [40] M. Nilsson, The DOSY Toolbox: A new tool for processing PFG NMR diffusion data, J Magn Res, 200 (2009) 296-302.

[41] D.S. Wishart, D. Tzur, C. Knox, R. Eisner, A.C. Guo, N. Young, D. Cheng, K. Jewell, D. Arndt, S. Sawhney, C. Fung, L. Nikolai, M. Lewis, M.A. Coutouly, I. Forsythe, P. Tang, S. Shrivastava, K. Jeroncic, P. Stothard, G. Amegbey, D. Block, D.D. Hau, J. Wagner, J. Miniaci, M. Clements, M. Gebremedhin, N. Guo, Y. Zhang, G.E. Duggan, G.D. MacInnis, A.M. Weljie, R. Dowlatabadi, F. Bamforth, D. Clive, R. Greiner, L. Li, T. Marrie, B.D. Sykes, H.J. Vogel, L. Querengesser, HMDB: the human metabolome database, Nucleic Acids Research, 35 (2007) D521-D526. 